\title{
Challenging Cases in Urothelial Cancer: Case 23
}

\author{
Mark S. Soloway ${ }^{\mathrm{a}, *}$ and Neil A. Abrahams ${ }^{\mathrm{b}}$ \\ ${ }^{a}$ Urologic Oncology, Memorial Physician Group, Division of Urology, Memorial Healthcare System, Aventura, \\ FL, USA \\ ${ }^{\mathrm{b}}$ Department of Pathology, Memorial Healthcare System, Aventura, FL, USA
}

Received 22 November 2021

Accepted 1 December 2021

Pre-press 20 December 2021

Published 11 March 2022

This is a 69 year old healthy woman who has a long history of urothelial cancer of the bladder. She was a cigarette smoker from age 20 to 35 when she stopped. She smoked one pack per day. She has had an appendectomy and a total abdominal hysterectomy.

Her first bladder tumor was discovered in 2008 after an episode of gross hematuria. A CT scan of the abdomen and pelvis was normal. She underwent a transurethral resection (TUR BT) and the pathology indicated a low grade noninvasive papillary urothelial carcinoma (Ta). Subsequent three to six monthly follow up office cystoscopies were normal until 2010 when she had a small papillary low grade Ta appearing tumor which was treated with office cautery. Over the next six years she either had no tumor or small papillary low grade appearing tumors which either were cauterized in the office or monitored, i.e., active surveillance.

In May 2016 two small i.e., $<0.5 \mathrm{~cm}$, low grade appearing Ta tumors were seen and were monitored until September 2016 when they were cauterized in the office. In January 2017 very small tumors were again seen on the left bladder and were removed in July 2017. A formal TUR BT was performed at this

${ }^{*}$ Correspondence to: Mark S. Soloway, MD, Chief, Urologic Oncology, Memorial Physician Group, Division of Urology, Memorial Healthcare System, Aventura, FL, USA. E-mail: mssoloway@yahoo.com. time and the pathology was high grade (grade 2) Ta. In March 2018 endoscopy indicated erythema on the left bladder wall and cold cup biopsies followed by resection and cauterization was performed. Figures 1 and 2. The pathology revealed carcinoma in situ (CIS). Figures 3 and 4. She received six weeks of intravesical BCG.

She remained free of bladder cancer until February 2019 when there was an area on the left wall which was suspicious for CIS. Urine cytology was suspicious for cancer. Endoscopic biopsies under anesthesia were performed. There was no bladder cancer.

In September 2020 small tumors were identified during a three-monthly office endoscopy. A TUR BT revealed high grade Ta urothelial cancer. The tumors were quite small and were removed with a cold cup biopsy forceps to avoid cautery artifact. The area was then cauterized. This was again followed with a sixweek course of BCG.

Her next endoscopy in January 2021 revealed a small area of erythema again located at the left lateral wall. Biopsies revealed focal CIS. She had a left distal ureteral stone three weeks later which required endoscopic removal. The CT scan performed showed normal upper urinary tract with the except of the calculous. Two weeks later she began another six-week course of BCG. In May 2021 she was found to have a broad 3-4 cm erythematous area in the posterior bladder toward the left wall. Figure 5. Urine cytology 


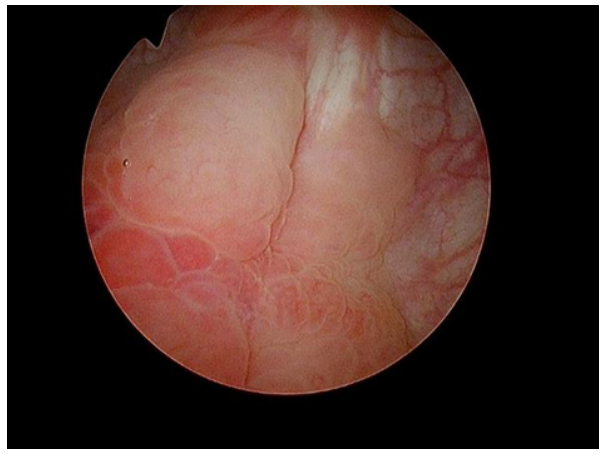

Fig. 1.

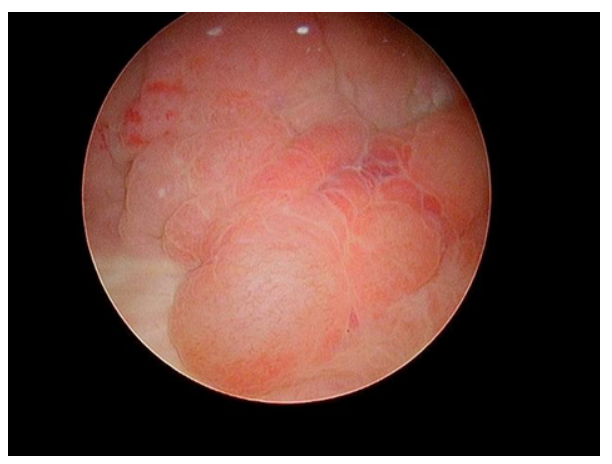

Fig. 2 .

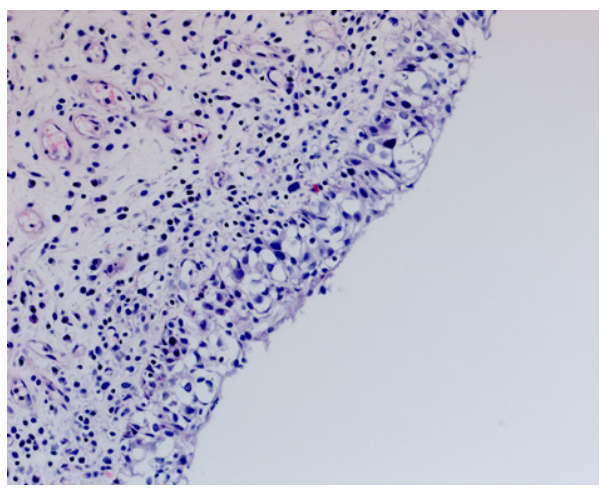

Fig. 3.

yielded high grade cancer cells. Figure 6. The area was initially sampled with a cold cup biopsy forceps before resection with the bipolar resectoscope. This area was cauterized with the bipolar button. The pathology was high grade urothelial cancer with a small focus of lamina propria invasion. Figure 7. There was no muscle in the specimen.

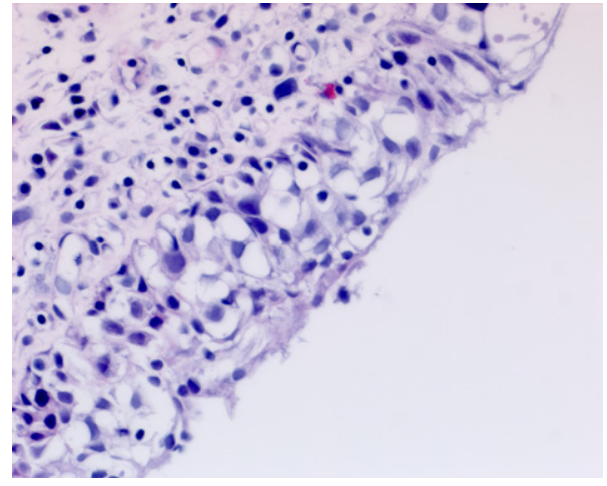

Fig. 4.

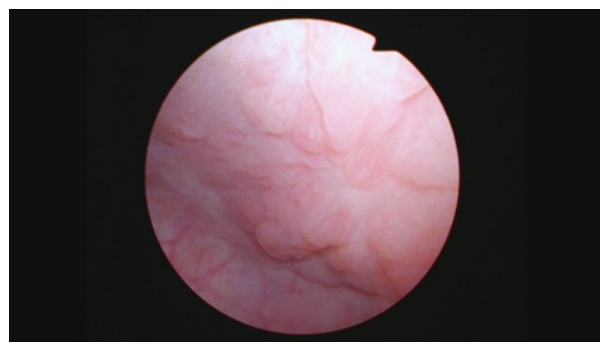

Fig. 5.

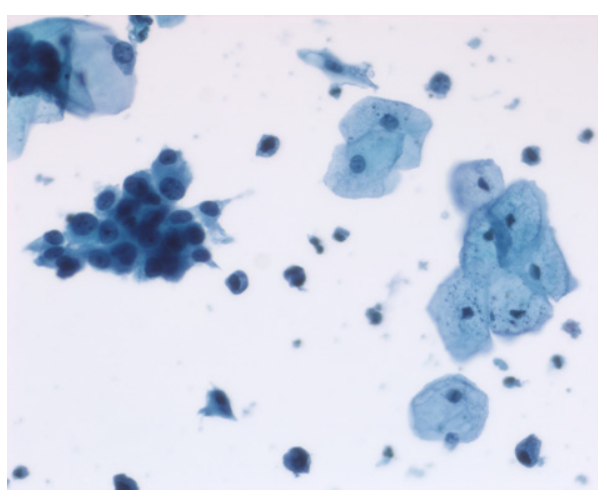

Fig. 6.

I had a lengthy discussion with the patient and reviewed the prior and current pathology reports. She had no voiding complaints. I discussed radical cystectomy, another course of BCG and also the encouraging results of pembrolizumab for patients with "recurrent" HG Ta/CIS/T1 urothelial cancer despite BCG.

She wanted to avoid a cystectomy, if possible, and agreed to receive pembrolizumab, a PD 1 inhibitor. She took four courses and had no side effects. 


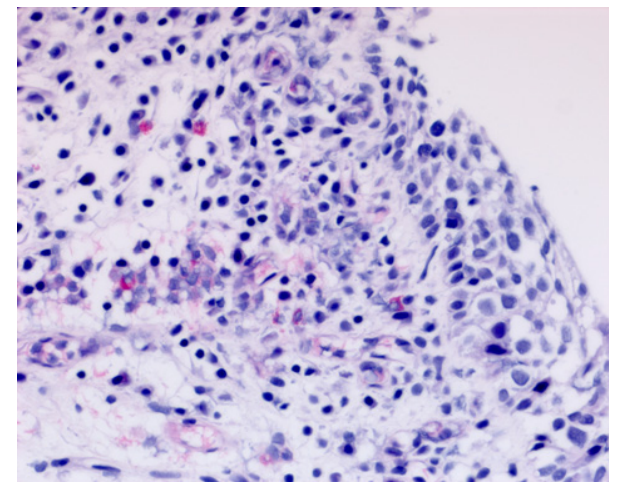

Fig. 7.

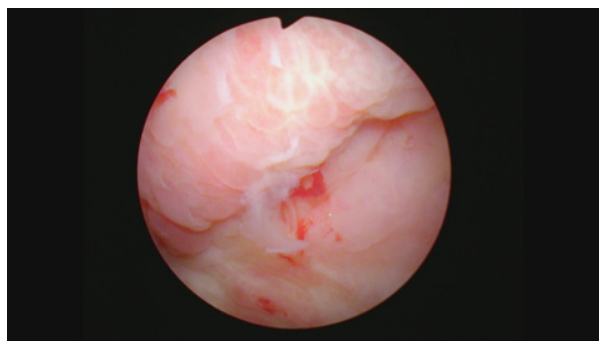

Fig. 8.

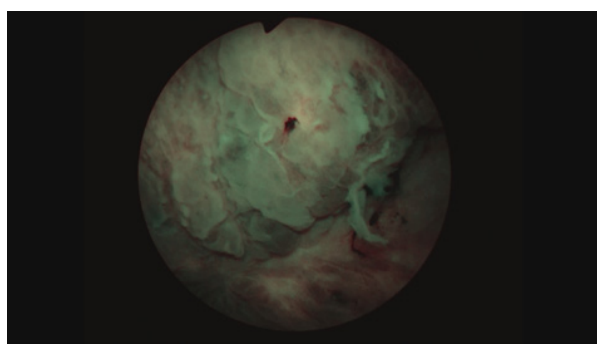

Fig. 9.

Cystoscopy in August 2021 indicated an erythematous patch on the left upper wall. Figures 8 and 9 . A TUR BT was performed, and the pathology was CIS with focal high-grade Ta. The lamina propria and muscle were present and tumor free. The medical oncologist who prescribed and supervised the immunotherapy ordered a CT scan. To my complete surprise there was a $4 \mathrm{~cm}$ mass adjacent to the anterior bladder wall. Figure 10. This was interpreted by the radiologist as suspicious for an invasive bladder tumor.

Since this finding, if correct, would change her stage and thus the treatment algorithm, i.e., a muscle invasive cancer and thus neoadjuvant chemotherapy, I

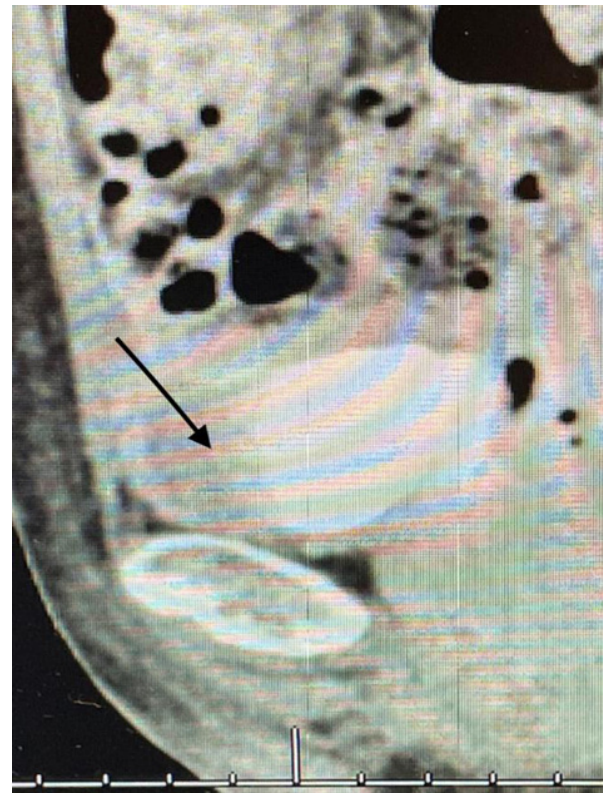

Fig. 10.

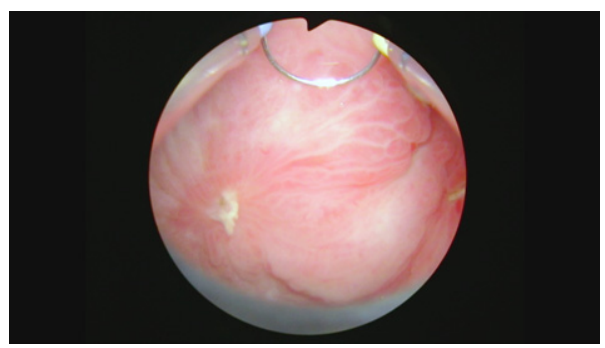

Fig. 11.

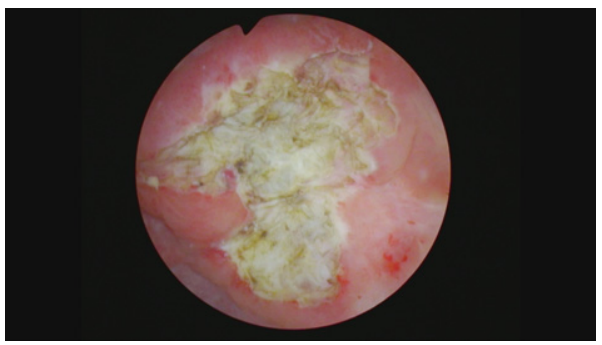

Fig. 12.

performed another TUR BT and resected the area that was contiguous with this "mass". I did not identify a mass. Figures 11 and 12. The pathology was CIS. A percutaneous biopsy of the area was performed by an interventional radiologist and there were no cancer cells. 


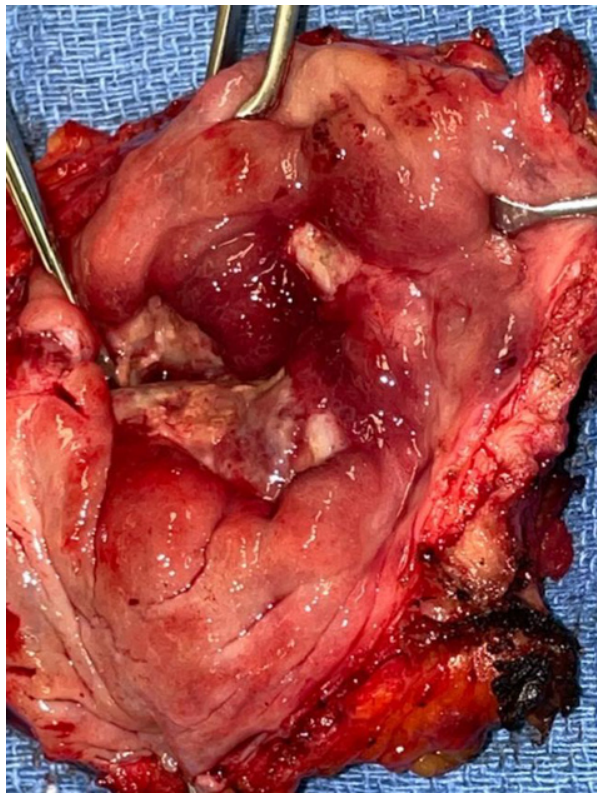

Fig. 13.

In October 2021 she consented to a total cystectomy. Figure 13. The vagina was preserved. An orthotopic neobladder was constructed. The final pathology was CIS. There was no mass adjacent to the bladder. I cannot account for the images on the CT scan.

This is not a typical case. Most men and women with initial low grade Ta bladder cancer never develop muscle invasive bladder cancer or even CIS. If they do develop a high grade T1 bladder tumor or CIS, it requires judgement and considerable interaction with the patient to find the ideal management strategy balancing the risk of developing a muscle invasive bladder cancer and the quality-of-life alteration with a cystectomy.
As the readers review this patient's course, I feel confident many of you have had similar patients with high grade Ta/T1/CIS bladder cancer who developed a new tumor despite BCG. When do you decide to abandon a bladder preserving approach and recommend a cystectomy? Have new intravesical agents or the PD1 and PDL1 inhibitors changed your management strategy?

\section{AUTHOR CONTRIBUTIONS}

MSS contributed to the work conception, performance, analysis of data and writing; NAA contributed to performance and analysis of data.

\section{INFORMED CONSENT}

Written informed consent for publication was obtained from the patient.

\section{ETHICAL CONSIDERATIONS}

The author has institutional review board approval for reviewing bladder cancer cases for outcome (MHS.2020.026).

\section{CONFLICT OF INTEREST}

MSS and NAA have nothing to disclose. 